\title{
Die letzten Masken. Zum Problem der Lüge bei
} Arthur Schnitzler

Die letzten Masken. Réflexions sur le problème du mensonge chez

Arthur Schnitzler

Last Masks. Reflexions on the problem of lying in Arthur Schnitzler's work

Gerhard Neumann

\section{CpenEdition}

\section{Journals}

Édition électronique

URL : http://journals.openedition.org/ceg/1795

DOI : $10.4000 /$ ceg. 1795

ISSN : 2605-8359

Éditeur

Presses Universitaires de Provence

Édition imprimée

Date de publication : 1 décembre 2014

Pagination : 185-194

ISSN : 0751-4239

\section{Référence électronique}

Gerhard Neumann, «Die letzten Masken. Zum Problem der Lüge bei Arthur Schnitzler », Cahiers

d'Études Germaniques [Online], 67 | 2014, Online erschienen am: 17 Dezember 2017, abgerufen am 04 November 2020. URL : http://journals.openedition.org/ceg/1795 ; DOI : https://doi.org/10.4000/ceg. 1795 


\title{
Die letzten Masken Zum Problem der Lüge bei Arthur Schnitzler
}

\author{
Gerhard NEUMANN \\ München und Berlin
}

\author{
Was ist Wahrheit? \\ Pilatus \\ Was sind Worte? \\ Konrad Herbot
}

Die Frage nach der kulturellen Funktion der Lüge gehört im Werk Arthur Schnitzlers zu den am häufigsten aufgeworfenen; und sie wird in der Regel mit einer anderen Frage in Verbindung gebracht: der Frage nach der Liebe, der Erotik nämlich und der mit dieser zusammenhängenden Thematik von Eifersucht, Seitensprung, Untreue und Betrug. Derjenige Text Schnitzlers, der diese thematische Konstellation von Lüge und Liebe vielleicht am differenziertesten vergegenwärtigt, ist der Einakter Die große Szene (1914/1915), der dem Zyklus Komödie der Worte angehört. ${ }^{1}$

Dieser Text soll mir zum Vorwand dienen, um das soziale Phänomen der Lüge, wie Schnitzler es auffasst, zu exponieren. In einem zweiten Schritt werde ich dann - im Vergleich mit diesem ,Satyrspiel' - wenn man es so nennen will - die eigentliche ,Tragödie' Die letzten Masken (1900/1901) ${ }^{2}$ einer Betrachtung unterziehen. Dabei soll deutlich werden, dass Schnitzler in seinen beiden Einaktern, einem „Experimentalgenre“, wie Hans-Peter

\footnotetext{
1 Arthur SCHNITZLER, Gesammelte Werke. Die dramatischen Werke, zweiter Band, Frankfurt am Main, S. Fischer Verlag, 1962, Große Szene, S. 491-529. Zitatbelege künftig nach dieser Ausgabe im laufenden Text.

${ }^{2}$ Arthur SCHNITZLER, Gesammelte Werke. Die dramatischen Werke, erster Band, Frankfurt am Main, 1962, Die letzten Masken, S. 719-735. Zitatbelege künftig nach dieser Ausgabe im laufenden Text.
} 
Bayerdörfer es genannt hat, das Problem der Lüge in den doppelten Kontext von Erotik und Tod gestellt hat. ${ }^{3}$

Zunächst also zum Einakter Die große Szene. Diese ist im Theater- und Schauspieler-Milieu angesiedelt und spielt in einem Hotelzimmer in Berlin direkt dem Theater gegenüber. Der Schauplatz des Geschehens ist also gewissermaßen exterritorial in der Grenzzone zwischen Privatheit und Öffentlichkeit angesiedelt, genau wie das Thema der Lüge, das in ihm verhandelt wird. Es sind Theaterleute, um die es geht; und um ihre Einstellung zum Kulturthema Lüge.

$\mathrm{Da}$ ist zunächst Dr. Falk, ein Theaterdirektor (in dem man Otto Brahm hatte erkennen wollen). Und da ist der geniale Schauspieler Konrad Herbot (als dessen Vorbild Josef Kainz gilt). Herbot, das Genie, ist in einer Schaffenskrise, weil seine Frau Sophie ihn verlassen hat.

Der Grund: Er hat sie, wie schon so oft, mit einer anderen Frau betrogen. Diesmal mit Daisy, einer Tochter aus gutem Hause und Braut eines gewissen Edgar Gley. Man hat Herbot nachts am Fenster Daisys beobachtet - und das Gerücht von seinem Ehebruch hat sich verbreitet und auch den Bräutigam erreicht.

Der Anfang des Stücks zeigt Sophie, seit deren Abwesenheit Herbot ,nicht mehr gut spielt'; die nun, um die Situation zu verbessern, auf Bitten des verzweifelten Dr. Falk, wieder zu ihrem Mann zurückgekehrt ist. Im Gespräch mit dem Theaterdirektor zeigt sie sich aber entschlossen, Herbot mehr zu verzeihen. Ihr Argument: er habe diesmal nicht mit einem ,Philinchen' (II, 501) Ehebruch begangen, wie des öfteren, sondern mit einer behüteten Tochter, einem unschuldigen Mädchen, einer jungen Braut, deren Leben nun zerstört sei. Das erstere sei entschuldbar gewesen, das letztere unverzeihlich und infam.

Ein Disput über den Stellenwert der Lüge in der Kultur entspinnt sich zwischen Sophie und dem Theaterdirektor. Dr. Falk plädiert für die Unschuld des Genies. Ein großer Künstler, in seinem Egoismus, wisse gar nicht, was er dem anderen antue. Und er versteigt sich zu der Behauptung: „Es gibt überhaupt keine Lüge auf der Welt. Es gibt nur Leute, die sich anschmieren lassen. Und zu denen haben Sie doch nie gehört.“ (II, 498) Und dann direkt an Sophie gerichtet: Es gebe nur eine ,unumstößliche Wahrheit', und das sei die Liebe (die zum Beispiel ihr Mann für sie empfinde). Dies führt ihn aber zugleich zu seiner These von den zwei ,Moralsystemen' zurück: Es gebe das Genie, das immer die Hauptrolle spielt; und es gebe die Betrogenen, denen das Recht bleibt, sich ihrerseits durch Untreue zu rächen.

Er spreche hier als, „unmoralischer Moraltheoretiker“ (II, 504), erklärt Dr. Falk und gibt sich so als Nietzsche-Leser zu erkennen. ${ }^{4}$ Die Genies seien

3 Vgl. Gerhard NEUMANN, Artikel „Einakter“ in Reallexikon der deutschen Literaturwissenschaft, Neubearbeitung des Reallexikons der deutschen Literaturgeschichte gemeinsam mit Harald FRICKE, Klaus GRUBMÜLLER und Jan-Dirk MÜLLER, herausgegeben von Klaus Weimar, Band 1 A-G, Berlin/ New York, De Gruyter, 1997, S. 409-422, hier S. $421 b$. 
stets von Abgangsapplaus begleitet, die Statisten stürben klanglos hinter der Szene. Sophie, angesichts des betrogenen Bräutigams, kommentiert denn auch: „Ja, sie haben schon etwas für sich, diese Menschen, die keine Genies sind, sondern ganz einfach brave Leute." (II, 505)

Nun verlässt Falk den Raum und das Ehepaar Sophie und Konrad Herbot beginnt mit der Aufarbeitung der Daisy-Geschichte. Herbot behauptet, er habe erwogen, dem betrogenen Bräutigam die Wahrheit zu sagen, um dem ,ewigen Lügenmüssen' zu entgehen. Er habe aber schließlich eingesehen, ,dass er es nicht tun durfte, schon wegen Daisys Familie' nicht (II, 511), Lügen also als Schonung der anderen. Sophie widerspricht zunächst. „Sei wahr“, beschwört sie ihn. „Ich könnte ja alles verstehen, alles verzeihen, nur um das eine fleh ich dich an, spiele keine Komödie“. (II, 512)

Und sie nimmt ihn dabei in Schutz mit Berufung auf das anthropologische Modell des guten Wesenskerns, der durch die Maske der kulturellen Rolle, die man spielt, nur verdeckt wird: „Was du da jetzt geredet, das warst ja nicht du. Es war manchmal ein Schein von dir, - der durch deine Maske leuchtet, aber du, du selbst, du warst es nicht." (II, 512)

In diesem Augenblick lässt sich Edgar Gley, der Bräutigam Daisys, melden. Sophie erschrickt: Hat Daisy ihm vielleicht alles gestanden? Und sie schwenkt auf die Position ihres Mannes ein. Ja sie nimmt, als Betrogene, Partei für das ehebrecherische Paar. „Ihr wart unvorsichtig, gewiß“, sagt sie. „Du bist durchs Fenster eingestiegen in der Nacht. Man hat dich gesehen. -“ „Er nicht““, stellt Herbot klar, „sonst wäre er nicht erst heute da.“ (II, 513) Da wird Sophie zur Komplizin: „Einmal noch darfst du - mußt du lügen“, gesteht sie ihm zu.

Herbot schickt Sophie aus dem Zimmer und lässt Edgar Gley eintreten. Dieser fällt mit der Tür ins Haus: „Ich habe eine Frage an Sie zu stellen, Herr Herbot. Antworten Sie einfach mit Ja oder Nein. Waren Sie Daisys Geliebter?“ (II, 514) „Herr Gley, ich bin fassungslos“ (II, 514), erklärt der Schauspieler - und lässt, ganz gegen diese Behauptung, alsbald ein SprachFeuerwerk von Lügen und halben Wahrheiten aufsprühen.

Er erklärt sich bereit zu einem Duell. Aber Gley insistiert auf dem Bekenntnis der Wahrheit: „Wir wollen hier keine Szene mit großen Worten spielen, Herr Herbot, wir wollen [...] miteinander reden wie zwei Männer [...], wie zwei Menschen [...], begreifen Sie endlich, daß hier ein Mensch vor Ihnen steht, Herr Herbot, der nichts anderes verlangt, als die Wahrheit, die Wahrheit, wie immer sie laute." (II, 515) Alles sei er zu ertragen imstande, nur nicht den Zweifel; alles könne er verzeihen, nur die Lüge nicht. Nicht als Rächer, nicht als Geck komme er, sondern als Mensch zu einem Menschen. Und Gley besteht darauf: „Die Wahrheit! Die Wahrheit!“ Und Konrad Herbot, gleichsam Pilatus parodierend, erwidert: „Was sind Worte?“ (II, 516)

\footnotetext{
${ }^{4}$ Nietzsches Text „Über Wahrheit und Lüge im außermoralischen Sinne“, 1883 entstanden, ist in der von Peter Gast besorgten Ausgabe aus dem Nachlass 1903 veröffentlicht worden. Friedrich NiETZSCHE, Werke in drei Bänden, dritter Band, hrsg. von Karl SCHLECHTA, München, Hanser, 1956, S. 309-322.
} 
Und hierauf wiederum Herbot: „Menschenskind, wie einfach sehen Sie noch die Welt! Ja und Nein! Und Wahrheit und Lüge! Und Treue und Untreue! - Wenn es so einfach wäre, junger Fr - Herr Gley" korrigiert er seinen gönnerhaften Versprecher. (II, 517)

Zuletzt holt Herbot einen Brief Daisys vom Tag seiner Abreise aus der Tasche, in dem sie ihn abweist und fortschickt. Durch Herbots Rede und schließlich durch den Brief, den Herbot verliest, zeigt sich Edgar von der Unschuld des Schauspielers überzeugt. Er wirft den Brief nach genauer Prüfung ins Feuer und zieht sich zurück.

Da taucht Sophie wieder auf. Sie hat die ,große Szene' Herbots vom Nebenzimmer aus verfolgt. „Jetzt kann ich dir's ja gestehen“, sagt Herbot und charakterisiert damit die Szene einzig und allein als schauspielerisches Virtuosenstück, zuerst war ich auch recht schwach, „ich hab ein wenig Lampenfieber gehabt im Anfang, trotzdem ich nicht ganz unvorbereitet war" (II, 522) - aber danach sei er zu großer Form aufgelaufen. Sophie stimmt ihm zu. Sie habe es sich wohl anders vorgestellt, fügt er hinzu: „Du hast dir gedacht, dass ich alles ableugnen werde.“ - „Und lügen“, fügt sie hinzu. „Lügen? Nein, Sophie. „Nur Dummköpfe leugnen.“ (II, 522) Und er fährt fort:

Nein, Sophie, es war nicht ausschließlich Lüge, es war mancherlei Wahres dabei. Das war gerade das Köstliche, wie es durcheinander gemengt war, das Wahre und das Falsche. Dadurch wurde es so absolut wahrscheinlich. Na, Gott sei Dank, jetzt kann man wieder ruhig atmen. (II, 522)

Nun stellt sich heraus: Das Datum des Briefes von Daisy war gefälscht. Es war der Anfangsbrief Daisys, den Herbot als Abschlussbrief zum besten gab. „Und der Brief - ich hab ihn schön gelesen, was? Der Brief, der in Wirklichkeit die Sache natürlich erst zum Klappen brachte, der war geradezu wie geschaffen, uns - wie soll ich sagen - als ein Alibi zu dienen.“ (II, 523)

Und Herbot beteuert noch einmal den Kunstcharakter der ganzen Szene: „Es gab Momente, in denen ich so mitgerissen war - es hat nicht viel gefehlt und ich hätte die ganze Geschichte selber geglaubt.“ (II, 523)

„Nein. Es fängt nicht an, es hört auf“, erklärt Sophie dem wieder erschienenen Dr. Falk. „Endgültig hört es auf [...] Ich reise ab“(II, 524) Das Ganze habe sie mit Ekel erfüllt: „Daß ein Mensch so lügen kann, das hab ich nie geahnt.“ (II, 524) Und wieder revidiert sie zuletzt ihren TrennungsEntschluss um des Genies ihres Mannes Herbot willen. Als dieser erklärt, ohne ihre Anwesenheit in der Loge werde er nicht spielen, und vor ihr niederkniet und sie fragt: „Kommst du?" antwortet Sophie zwar nicht; aber es heißt in der Bühnenanweisung: ,nur ihre Miene drückt ihre Zustimmung aus.“ (II, 528) Das Paar verlässt das Hotel in Richtung Theater. 
$\mathrm{Zu}$ diesem Text ist einiges zu sagen. Dieser Teil der Szene, die ich jetzt skizziert habe, bildet das Herzstück des Einakters, es ist die Inszenierung einer Theorie der Lüge und ihrer Rolle im kulturellen Feld. Dabei ist eine ganze Reihe von Beobachtungen hervorzuheben.

$\mathrm{Da}$ ist zunächst die Zwiespältigkeit des Begriffs der Lüge: ,Lüge ist nicht Lüge', heißt es da. Für das Genie und den Durchschnittsmenschen gelten nicht die gleichen Regeln. Das Genie - in diesem Fall der Schauspieler - ist ein Virtuose der Lüge. In seinem Mund, in seinem Körperausdruck ist sie nicht mehr ein moralisches, sie wird zum ästhetischen Phänomen: sie wird zur kunstvollen Lösung eines Dilemmas! Ein Drittes sodann: Die Szene zeigt Spuren der Nietzscheschen Theorie von der Lüge, wie er sie in seinem Aufsatz „Über Wahrheit und Lüge im außermoralischen Sinne“ entwickelt hat. Es ist die Auffassung, dass der Mensch nur Sprache, nur Metaphern besitzt und keinen Zugang zur Wahrheit hat, mithin sein reden auch nicht als lügen verwerflich sein kann.

„Wir wissen immer noch nicht, woher der Trieb zur Wahrheit stammt“, schreibt Nietzsche. „Denn bis jetzt haben wir nur von der Verpflichtung gehört [...], nach einer festen Konvention zu lügen, herdenweise in einem für alle verbindlichen Stile zu lügen. ${ }^{55}$ Nietzsches Sprachrohr ist Dr. Falk, der sich selbst als ,unmoralischen Moraltheoretiker' bezeichnet.

Eine vierte These ist zu formulieren: Lüge erweist sich als ein Problem und notwendiges Element des Rollenspiels, als das Schnitzler jede soziale Kommunikation angelegt sieht. Es ist das Problem einer unausweichlichen theatralen Struktur in der Kultur, in der das Phänomen Lüge als Motiv und Triebfeder des Handelns notwendige Bedingung ist. Dies wird, als ein weiteres Argument, unter dem Aspekt der Lüge in der Paarbeziehung, genauer markiert: Komplizenschaft, die in der sozialen Rolle begründet ist, reicht weit über persönliche Kränkung hinaus.

Ein letzter, besonders wichtiger Punkt betrifft aber dann die Machtspiele, die beim souveränen Verfügen über den Diskurs, aber auch beim Spielen und Zusammenspielen verschiedener Diskurse, Anwendung finden - zum Schaden der Schwächeren und weniger Erfolgreichen; der ,braven', wie Sophie sie nennt. „Wir Komödianten“, sagt Herbot an einer Stelle und beruft damit ein elitäres ästhetisches Modell, das in das Leben der Kultur und ihrer Wertewelt einwirkt. In der Großen Szene wird dieses Verhalten zum Erfolgsmodell. In den Letzten Masken, die im Folgenden zu behandeln sind, erweist es sich als Muster des Versagens, der Wirkungslosigkeit: ob Lüge oder Wahrheit, das ist nicht mehr relevant. Aus anderen Gründen als denen im Erfolgsmodell, wie zu zeigen sein wird.

Das Kernstück des Einakters Die große Szene ist jene Situation, in der es Konrad Herbot durch Anzettelung einer ,Komödie der Worte' gelingt, den

${ }^{5}$ Ibid., S. 314. 
betrogenen Bräutigam von seines, des Schauspielers Herbot, Unschuld zu überzeugen. Dies geschieht durch das geschickte, von Herbot virtuos gehandhabte Inszenieren und Überblenden verschiedener Diskurse. Der gefälschte Liebesbrief, den Herbot vorweist und der im Grunde dem sexuellen Diskurs zwischen ihm selbst und Daisy, zwischen Verführer und Verführter entspringt, dient ihm als Libretto eines empfindsamen Gegendiskurses zu diesem. Das corpus delicti wird so gewissermaßen verschüttet und mutiert, wie Herbot selbst sagt, zu seinem Gegenteil, einem alibi. Wo Herbot dem Bräutigam ,in Wahrheit' das ius primae noctis raubt, wird, ,in der Lüge', der Schleier der Unberührtheit über das sexuelle Faktum gelegt. Dies geschieht durch ein virtuoses doppelzüngiges Umcodieren des sexuellen Diskurses in einen empfindsamen Seelen-Diskurs: Dies ist die ,große Szene', die Herbots genialer Strategie entspringt: „,ich war nicht ganz unvorbereitet"! Daisy wird in die Unschuld zurückdefiniert. Zwischen Herbot und Gley findet, von dem Schauspieler inszeniert, ein Kunstakt statt, der die fatale Situation durch virtuoses Reden bereinigt, den Künstler Herbot eine „Männerhochzeit“ (Wilhelm Raabe) mit Gley zelebrieren lässt. Die Frauen sind aus der Situation herausgedrängt: entweder als Zeugin, wie Sophie, oder als - freilich frivoles - Opfer dieses Vorgangs, wie die Braut Daisy. Das Argument der Leidenschaft dient paradoxerweise dazu, das Sexuelle zu verdecken. Es ist eine kunstvolle Strategie der Zweckentfremdung des empfindsamen Diskurses - eine ,Komödie der Worte'. Es mag diese in der Ambivalenz der Sprache begründete Diskursverfälschung und die aus ihr herausinszenierte skrupellose Frivolität gewesen sein, die Schnitzler für seine Zeitgenossen so anstößig machte, dass sie ihn, anlässlich des Reigens, vor Gericht zitierte.

\section{III}

Schnitzlers Einakter sind Experimente auf exemplarische Fälle, ähnlich Mussets Proverbes dramatiques. Schnitzler arrangiert sie gern in Zyklen, die sie ins Licht einer kulturellen Leitvorstellung versetzen: in den von mir gegebenen Beispielen ins Zeichen der Lüge. So wie die Große Szene im Zyklus mit dem Titel Komödie der Worte steht, so erscheint der Einakter Die letzten Masken unter dem Thema Lebendige Stunden. Wie der gleichnamige Text, nämlich der Einakter Lebendige Stunden, deutlich macht, versteht Schnitzler darunter ein Aufscheinen der Lebenskraft, des authentischen, vitalen amoralischen Lebenswillens, jenseits der Differenz von Wahrheit und Lüge.

Ist der Einakter Die große Szene eine Auseinandersetzung um Liebe, Lüge und Wahrheit, so das Stück Die letzten Masken eine solche um den Hass, den Tod und die angesichts seiner noch mögliche oder unmögliche Option für das, was man die ,Wahrheit' nennt. 
Der erfolg- wie mittellose Journalist Karl Rademacher liegt im Sterben; in der Klinik, in welcher Dr. Halmschlöger Stationsarzt ist. Rademacher sitzt im sogenannten „Extrakammerl“ und dämmert dem Tod entgegen. Die erste Person, die auf der Bühne zu Wort kommt, ist der Schauspieler Florian Jackwerth, wie Rademacher dem Tod geweiht; er weiß es nur noch nicht. Was ihn als Schauspieler brennend interessiert, ist die Wahrnehmung und Darstellung des Todes. Er beobachtet die Sterbenden in der Klinik und erschreckt die Nachtschwester, indem er die Grimassen der Moribunden nachspielt. Als er sich über Rademacher beugt, um ihn zu studieren, ist er unsicher: „Meiner Seel', ich hab' gemeint, er ist schon tot!““ (I, 719)

Aber Rademacher öffnet die Augen und Jackwerth gesteht ihm: „Ich hab' einfach allen Leuten, auf die ich einen Zorn gehabt hab', innerlich die fürchterlichsten Grobheiten g'sagt. Oh, das erleichtert, das erleichtert, sag' ich Ihnen!“ (I, 722)

Inzwischen ist Visite. Rademacher ist stark erregt. Er habe noch einen Wunsch, erklärt er dem Arzt: Er wolle seinen Jungendfreund noch einmal wiedersehen, den erfolgreichen und begüterten Alexander Weihgast. Es stellt sich heraus, dass Dr. Halmschlöger den berühmten Dichter persönlich kennt. Er erklärt sich bereit, ihn zu holen. „Meiner Ansicht nach sind gerade solche Dinge das Allerinteressanteste in unserm Beruf" (I, 726: zugleich die Stimme des Autors Schnitzler!).

Jackwerth fragt Rademacher nach dem Grund für seinen letzten Wunsch. Rademachers Antwort: Er habe einen tödlichen Hass auf Weihgast, der immer sein Rivale war und alles erreicht habe, was ihm, Rademacher, versagt geblieben sei. Er habe aus Rache Weihgasts Frau verführt und wolle ihm vor seinem Tod diese ganze Wahrheit ins Gesicht schleudern; ihn dadurch regelrecht vernichten. Es sei, so bekennt er, Jackwerth gewesen, der ihn auf die Idee mit der erleichternden Hass-Rede gebracht habe. Und der Schauspieler hat einen Einfall: Rademacher solle doch die Hass-Rede proben, bevor er sie ,in Wahrheit' halte. Er, Jackwerth, werde die Rolle des Rivalen Weihgast spielen; er sei vorzüglich, gerade im Extemporieren. Die Probe gelingt. Jackwerth schmettert pflichtschuldig: „Ha, Elender, du lügst!“ Die Hassorgie Rademachers zeigt sich so vollkommen wie es Herbots Redeschwall in der Großen Szene war, mit dem er den betrogenen Bräutigam Gley über die Wahrheit hinwegtäuschte.

Weihgast kommt, die beiden sprechen miteinander. Rademacher erkundigt sich höflich nach Weihgasts Gemahlin und den Kindern. (I, 732) Er fügt ein Cliché an das andere - „So wird man alt, und das Leben nimmt seinen Lauf“" (I, 733) - und verabschiedet sich zuletzt von Weihgast mit den Worten: „Ich hab' dich nur noch einmal sehen wollen, mein alter Freund, - das ist alles. Ja." Eine Lüge zuletzt. Er hat die ,Wahrheit' nicht über die Lippen gebracht. Rademacher zu Jackwerth: „Was hat unsereiner mit den Leuten zu schaffen, die morgen noch auf der Welt sein werden?" (I, 735)

Die letzten Masken ist ein Experiment auf die Lüge angesichts des Todes. Wie die Große Szene die Virtuosität der Lüge zeigt, die als Kunstwerk den 
Triumph der Erfindung sogar über den Redenden und sein Besser-Wissen erzielt, so bleibt die Wahrheit, die im Angesicht des Todes, wie die gängige Meinung ist, durch nichts mehr aufgehalten werden kann, ungesagt. Die Differenz zwischen Wahrheit und Lüge ist erloschen.

Beide Male sind es also Inszenierungen, die stattfinden, ein unausrottbares Insistieren auf dem Theater, als welches das Leben gespielt wird; die Grenze zwischen Wahrheit und Lüge verwischend, die moralischen Unterscheidungen auflösend: einmal im Zeichen der genialen Schauspielkunst, das andere Mal im Zeichen der Gleich-Gültigkeit des Lebens im Todesaugenblick.

\section{IV}

Was die beiden Einakter, um die es hier geht, verbindet, ist, dass jeder von ihnen um das Theaterritual der Probe kreist. Herbot probt nicht, aber ist gut vorbereitet, improvisiert und erzielt einen gelungenen Auftritt. Rademacher macht eine Probe, um für das Gelingen vorbereitet zu sein, und verzichtet zuletzt auf die Aufführung. Die Probe markiert die Differenz zwischen Wahrheit und Lüge, zwischen Wahrheit und Erfindung.

Dem genialen Herbot dient die Improvisation (und das sie stützende richtige Requisit, der Brief) zur Erreichung seines Ziels, nämlich der Umcodierung der erotischen Rede in eine empfindsame. Rademacher wird von einem Schmierenkomödianten eine Probe angedient, welche durch ihr Gelingen seiner Rache-Rede die ,Wahrheit' sichern soll. Aber er ist nicht professionell genug, diese Rede als ,gelernte' im Ernstfall zu reproduzieren. Und er ist als Sterbender nicht in der Lage, ihr moralisches Kapital auszumünzen.

Was die Gegenüberstellung der beiden Einakter aus sich entlässt, ist das Repertoire aller möglichen Auffassung von dem, was ,Lüge' ist und sein kann. Diese reichen von der Infamie der Lüge bis zur Wohltat der schonenden Unwahrheit, der Empathie des Verschweigens. Einen Fächer dieser Möglichkeiten könnte man öffnen, aber dann auch gleich wieder schließen.

Erstens: Lüge, wenn sie etwas Kostbares zerstört, ist infam. Es ist ein Standpunkt, den Sophie vertritt.

Zweitens: Von maßgeblicher Bedeutung ist die - außerhalb der Moral gestellte - Frage, wer es ist, der lügt; also die Qualität des Lügners. Es gibt im Feld der Lüge hierbei zwei Positionen: einerseits die des Genies, das durch virtuose Strategien sein Ziel - mag es gut oder böse sein - erreicht; andererseits die des ,Angeschmierten', der die Wahrheit nicht verdient.

Drittens: Der Umgang mit der Lüge ist in besonderer Weise das Tun des Schauspielers. Durch geschicktes Manipulieren gelingt es ihm, Diskurse in ihr Gegenteil zu verwandeln, sogenannte ,Lüge' in sogenannte ,Wahrheit' zu übersetzen. Die perfekte Intrige kann so weit führen, dass der ,Lügende' seine 
eigene Geschichte für wahr hält. Es ist ein Musterfall der Selbstüberredung. (Konrad Herbot) Eigentlich sind wir alle Schauspieler.

Viertens: In der Nachfolge Nietzsches gibt es den ,unmoralischen Moraltheoretiker', wie ihn Dr. Falk vertritt. Er hat die Lüge aus dem ethischen Feld herausgenommen, betrachtet sie aber in kulturkritischer Perspektive. In dieser zwiespältigen theoretischen Position unterscheidet er sich von jenen Theoretikern, die sich auf die Virtuosität der Lüge konzentrieren und diese allein im ästhetischen Feld ansiedeln.

Fünftens: Daraus leitet sich ein weiterer Fall ab. Es ist der Standpunkt des Rhetorikers unter den Lügnern. Er stellt - mit Herbot - die Frage: ,Was sind Worte' und insistiert auf der Ambiguität der Sprache, die bei geschickter Behandlung Wahres und Falsches mischt und dadurch das beliebig ,Wahrscheinliche' plausibel macht.

Sechstens: Noch einen Schritt weiter geht die antike Überlieferung, die im Sinne Platons Dichtung als Lüge verwirft. Hier wird auch das Argument der Metapher als unzuverlässiger Mimesis wirksam: eine Frage, die schon Aristoteles bei der Erörterung vom Menschen als dem Wesen stellt, das gerne nachahmt, und dem Wesen, das Lust am Ähnlichen hat. Die negative Seite dieses Gedankens - Nachahmung ist nicht das Original und Ähnlichkeit ist nicht Identität - wird dann zur Voraussetzung von Nietzsches Diffamierung der Metapher.

Siebtens: Ein weiterer Punkt ist die Funktion der Lüge im Feld der Lebensarchitektur, des Aufbaus einer Karriere des Subjekts und der Krisen, die sich daraus ergeben: Goethe hat dies benannt, im Titel seiner Autobiographie. Hier hat die Lüge eine ästhetische, eine ethische, und eine empathische Funktion. Dies gilt namentlich auch für Biographien von Hochstaplern, wo die Lüge zum Inspirationsorgan wird. (Felix Krull)

Achtens: Eine wichtige Position nimmt die Lüge im Kontext des ,sich etwas von der Seele Redens' ein. Dies geschieht durch Aufhebung der Lüge so gut wie durch neue, erfundene, die Situation bessernde Lügen, die so eine diätetische Funktion gewinnen. (Es ist die Position des schlechten Schauspielers Florian Jackwerth und in der Folge diejenige Rademachers.)

Neuntens: Die Lüge ist ein Mittel der Schonung, des Mitleids, der (ärztlichen) Menschenfreundlichkeit - vielleicht auch der Bequemlichkeit. Schnitzler hat mit Bedacht das Sterbezimmer einer Klinik zum Ort solcher schonenden Lügen gemacht.

Zehntens: Zuletzt sind diejenigen - als äußerstes Ende der Skala - zu nennen, die stricte auf Lügen verzichten, die Wahrheit zur Lebensform erheben, ,in ihr wohnen': Sophie nennt sie die ,einfach Braven' und der betrogene Bräutigam Gley verkörpert diese Spezies.

Hinter all diesen aufgesplitterten Varianten der Lüge stehen in der Moderne wohl zwei deutlich unterscheidbare Theorien. Die erste fußt auf der Auffassung von der Metaphorizität der menschlichen Sprache, ihrer 
fortgesetzten, nicht arretierbaren Verschiebung der Referenz. ${ }^{6}$ Hier gibt es keine Instanz, die auf die Dichotomie von Lüge und Wahrheit rekurrieren oder sie gar sichern könnte. Die zweite Theorie nimmt Bezug auf ein anthropologisches Faktum, den unbändigen Drang des Menschen nach Inszenierung. ${ }^{7}$ Man hat dies das Argument von der Theatralisierung des Lebens genannt. Das Drama wäre dann, wie schon Aristoteles glaubhaft macht, die Urform der Kommunikation, der Mensch als Schauspieler ihr Protagonist.

Auf beide Theorien nimmt Schnitzler implizit Bezug, in beide Traditionen stellt er seine Texte hinein: die Idee von der Theatralität und die Idee von der Ambiguität der Sprache.

Wollte man eine Formel für Schnitzlers Umgang mit der Lüge finden, so müsste man sie im Feld der Ästhetik suchen, nicht der Ethik. Am lakonischsten ist eine solche Formel wohl Giuseppe Verdi gelungen. In einem Brief an Clara Maffei vom 20. Oktober 1876 schreibt er: „Die Wahrheit nachzuahmen mag eine gute Sache sein; aber es ist noch viel besser, wenn man die Wahrheit erfindet.“

\footnotetext{
${ }^{6}$ Jaques Derridas Konzept der déconstruction und der différance beruht auf dieser Annahme.

${ }^{7}$ Roland Barthes Kulturtheorie beruht im wesentlichen auf einer solchen Vorstellung der Inszenierung. Vgl. sein Buch $S / Z$, in dem er eine Novelle Balzacs unter dem Gesichtspunkt ubiquitärer Inszenierungen von der Geschlechterrolle bis zum Kunstakt analysiert: Roland BARTHES, $S / Z$, Paris, Éd. du Seuil, 1986
} 